\title{
Object Isolation with Minimal Impact towards the Object of Interest in a Complex Environment Using Manipulation Primitives
}

\author{
Quah Jit Shen, Muhammad Fahmi Miskon, Khalil Azha Mohd Annuar
}

\begin{abstract}
It is common in the field of robotic manipulation to specifically target and precisely move, displace or manipulate the targeted object of interest. This however may not always be the best possible course of action as there are situations where it is not possible to manipulate the object of interest or is not in a condition to be manipulated. This research paper explores and subsequently proposes 3 object isolation technique for the purpose of isolating a targeted object of interest from the environment incontras to the standard utilization of object singulation technique. The goal was to develop an algorithm than can successfully isolate the object of interest from the environment via removing the environment without/with minimal impact towards the object of interest. Results from the experiment indicated that the proposed algorithms can successfully isolate the object of interest with minimal impact towards the object of interest scoring an average of $0.85 \mathrm{~cm} /$ actuation for MSMAPPS, $0.75 \mathrm{~cm} /$ actuation for MSMAPOS and finally $0.27 \mathrm{~cm} /$ actuation for BSMAPOS. These results indicates a relatively small displacement per actuation at 4.35\% displacement per actuation, $3.75 \%$ displacement per actuation, and $1.35 \%$ displacement per actuation relative to the workspace respectively.
\end{abstract} Object Isolation

\section{INTRODUCTION}

T he research paper is a follow up of a previous research which looks into the usage of isolation techniques instead of singulation techniques for the purpose of isolating the environment from the object of interest [8]. In this research, three novel novel algorithm which exploits the use of simple manipulation primitives to completely isolate an object through indirect manipulation of its the environment was proposed. Through experimentation and past results, our method is capable of successful isolation with minimal impact towards the object of interest. We demonstrated the effectiveness of our single manipulation approach using a 5 DOF KUKA YouBot to isolate bricks of specific colour away from a single brick of interest with another colour.

Revised Manuscript Received on October 22, 2019.

* Correspondence Author

Quah Jit Shen*, Center of Excellence in Robotics and Automation, Faculty of Electrical Engineering, Universiti Teknikal Malaysia Melaka, Hang Tuah Jaya, 76100 Durian Tunggal, Melaka,. Email: quahjit@gmail.com

Muhammad Fahmi Miskon, Center of Excellence in Robotics and Automation, Faculty of Electrical Engineering, Universiti Teknikal Malaysia Melaka, Hang Tuah Jaya, Email: fahmimiskon@utem.edu.my

Khalil Azha Mohd Annuar, Faculty of Engineering Technology, Universiti Teknikal Malaysia Melaka, Hang Tuah Jaya, 76100 Durian Tunggal, Melaka, Malaysia, Email: khalilazha@utem.edu.my
Keywords: Trajectory Generation, Environment Manipulation,

Object isolation in essence is the problem of removing the clutter/unwanted objects away from the object of interest or the object of interest from the cluttered environment. Most research when it comes to isolation focuses on the removing the object of interest from the scene [1-8] as it is often the fastest way to isolate and arguably the most useful in the industry. Methods to tackle said problem in the manner mentioned previously often coincides or is in fact often treated as an extension to the field of object sorting due to its precise nature in handling the objects with minimal interactions to its surroundings.

However, there is a case to be made for the removal of clutter/unwanted objects from the object of interest as well. There're cases where we're unable to remove the object of interest because it is either too big or heavy to be manipulated. An example of such case would be the case of an exploratory robot wanting to collect training images of a interesting monolith but is blocked by environmental objects such as leaves, smaller manipulatable rocks and even people where if allowed to remain in the training image can lead to incorrect perception [1,9] and ultimately failure in future operations $[5,6,10-15]$. There are also cases where we might not want to manipulate the object of interest even if we could. Such a case is for example robots used the rescuing of victim after an earthquake or even robots used for archaeological excavations. In both cases, while it is possible to manipulate the victim/artefact from where they are found, we would not know if said person is injured or the artefact is brittle potentially causing more harm than good. Therefore, a better course of action would be to clear off the surrounding rubble so that experts can come in to access the victims or said artefact. The lack of literature for this specific issue combined with the low efficiency of current employed methods becomes a motivating factor for this research. Our past research has indicated it is possible to improve the efficiency in accomplishing the task of object isolation when using this 3 devised algorithms by a significant amount when compared to current utilized techniques. However, the performance in terms of the impact of indirect manipulation throughout the entire isolation process towards the object of interest has yet to be evaluated. While logic indicates that it would definitely be faster for us to clear the field with a sweaping motion aimed at the largest pile in the field, doing so might displace the object of interest indirectly and that might go against the idea of devising a task specific methodology to solve the issue as discussed previously. 


\section{Object Isolation with Minimal Impact towards the Object of Interest in a Complex Environment Using Manipulation Primitives}

This thus becomes a challenge when devising a method to isolate the object of interest which exploits the use of indirect manipulation to remove the environment while at the same time minimizing the same effects of indirect manipulation towards the object of interest itself.

\section{LITERATURE REVIEW}

As mentioned previously, object isolation is a niche field of study which stems from a larger group of study known as pile manipulation/ interaction [2-6]. The field of isolation itself can be broken down into two circumstances which are isolation where we aim to remove the environment from the object of interest and singulation where we remove the object of interest from the environment. However, current literature focuses on the utilization of object singulation techniques for both isolation and singulation circumstances. This is rightfully so as singulation techniques are precise and accurate in the sense that it only manipulates what it needs to manipulate while leaving the rest untouch. As of such, the manipulation primitives commonly employed for this is pick and place due to the requirements of precision in the act of relocating/repositioning the objects from a pile. This motion is often assisted by perturbing pushes to loosen/separate the objects that were in a pile to make it easier for grasping to occur $[2,5,6]$. By having small and discrete motion to adjust the individual object within the pile into a desirable configuration and subsequently manipulating it out with pick and place, the method boosted a high accuracy in manipulation however suffers in terms of number of manipulations required to clear off the field.

From issues raised by the previous research $[2,5,6]$, to reduce the number of manipulations required to clear off the field what some researchers did was that instead of manipulating a single object to improve the systems perception of it, the system instead manipulates the pile as a whole $[3,4]$. In this case, instead of poking and perturbing one object until it is reconfigured into an ideal position, they instead pushes/spread out/tumble the pile into a cluster of individual bricks followed by removing them one at a time using pick and place method. By doing this, they reduce the need to run multiple actions to improve perception onto a single object by doing it on multiple objects in a single move instead. This method successfully reduces the number of manipulations by a significant amount compared to the previous method by a significant amount with a small trade-off in terms of accuracy

Lastly, while all the other methods can successfully sort out the object of interest from the noise objects, most of them require numerous manipulations to remove a single object because they will take one action to improve its perception and another to reposition the object. To reduce the number of actions required for isolation, categorizing the noise object as a whole rather than its individual parts enables us to manipulate the pile/cluster of objects in a single action rather than multiple actions for a single object [7]. By using this method, it is possible to reduce the average number of manipulations to remove objects in a pile to less than one due to a single motion capable of removing multiple objects compared to the manipulation to improve perception followed

by the precise pick and place method mentioned previously [2-6]. However, this method has its own set of problems namely being only applicable on a very specific setup for example requiring multiple robots, robots with a specific end effector or utilization of tools.

While the methods mentioned previously manages to solve a lot of the problems involved within the field of isolation, the methods employed from [2-6] are mainly singulation techniques used for the purpose of sorting. While it is possible to apply singulation techniques in the problem of isolation, it is simply not as efficient. This inefficientcy is because when we apply singulation to the problem of isolation, singulation would regard all blocks in the field (environment) as important in contras to its usual method of regarding only the object of interest as important in the case of singulation. This in turn means that said isolation method will have to take multiple actions, first of which to precieve the object its trying to move and another to actually move the object to the desired position for each and every single object in the field one object at a time. Hence while it is possible to utilize individual object manipulation methods, it is simply inefficient when considering that we can remove multiple object at once directly using tools [7] or indirectly using manipulation casualties. This inefficiency can be seen in [6] where selecting and subsequently isolating each individual object takes a long time (6.6 manipulations for a single object) and said time would only multiply as the number of objects that it needs to manipulate is increased. Even in the situation where they did look into a multiple object manipulation [7], said method can only be applied with a very niche setup which is two robots and a rope tool hence making it not cross compatible across different kinds of robot platforms.

Our past work on object isolation has yielded that by using an isolation specific method, it is possible to solve the problems mentioned above in a simulated environment in a faster and hence more efficient method [8]. However, the performance of the system is not solely measured through its speed of actuation but also whenever if the algorithm has successfully fufill its intended goal which is to isolate the object through removing the scene without touching the object of interest due to specific circumstances such as fragility. In this regard, even if we could successfully remove the environment object via mass indirect object manipulation, we would still have failed our goal if throughout the process, the noise object constantly bumps and displace the object of interest hence disregarding the purpose of the predisposed specific circumstances earlier. This thus becomes the focus of this paper where we want to see if the developed method can successfully isolate with minimal affect towards the object of interest.

\section{ALGORITHM}

For this paper, we propose the use of single manipulation primitive (pushing movement) to isolate an object efficiently from a cluttered/complexed environment exploiting the effects of manipulation casualties to remove more than one noise object per manipulation hence reducing the overall time/manipulations required to fully isolate the object. At the same 
time, the designed algorithm has the capacity to be integrated on any mobile robot with any kind of end effector. In order to achieve the aforementioned goals, we developed and compared 3 novel algorithms which are the Mono-directional Single Manipulation Algorithm Using Pile Position Strategy (MSMAPPS), Mono-directional Single Manipulation Algorithm Using Pile Overlap Strategy (MSMAPOS) and Bi-directional Single Manipulation Algorithm Using Pile Overlap Strategy (BSMAPOS) to identify the most efficient of the three in comparison to the performance of Gupta et al's manipulation aided method [5]. All 3 algorithms comprise of 5 steps: 1) Object segmentation, 2) Pile Selection 3) Path Planning 4) Manipulation of region 5) repetition until the object of interest is completely isolated or the field is empty. The exact flow and its respective explanations are as follow:

\section{A. Object Segmentation}

- Objects are segmented into 2 distinct categories which are object of interest and noise objects.

\section{B. Pile Selection Strategy}

- For Pile positioning strategy, the noise object that is chosen to be manipulated is selected using a hierarchal methodology where the objects leftmost of the field is given priority followed by object that is furthest away from the mid-section of the field along the y axis. The operation is represented in the following algorithm:

$$
\begin{aligned}
& S P=\min (d(1), d(2), d(3) \ldots \ldots \ldots \ldots d(i)) \\
& \text { where } d(i)=\left[\left|y_{\text {noise }}-y_{\text {mid }}\right|+\left|x_{\text {noise }_{i}}-x_{\text {mid }}\right|\right]
\end{aligned}
$$

Given than SP denotes the selected pile, i denotes the total number of noise pile/object detected, $y_{\text {noises }}$ is the $\mathrm{y}$-coordinate of the noise object $\mathrm{i}, y_{\text {mid }}$ is the $\mathrm{y}$ coordinate for the centre of the workspace and $x_{\text {noise }}$ is the $\mathrm{x}$-coordinate of the noise pile/object $\mathrm{i}$.

For Pile Overlap strategy, the noise object to be selected is based on the pile whose identified border has the most overlaps with other piles border. In the event the number of overlap is equal then we revert back to pile positioning method to determine the pile to be manipulated. The operation is represented with the following algorithm:

$$
\begin{aligned}
& S P=\max (O(1), O(2), O(3) \ldots \ldots \ldots \ldots o(i)) \\
& \text { where } O(i)=\left[N_{\text {overlap }_{i}}\right]
\end{aligned}
$$

Given than $N_{\text {overlap }}$ denotes the number of overlaps for the corresponding noise pile/object i. Once the operation is complete, in both cases the $\chi_{S p}$ and $x_{S P}$ is forwarded to the next operation as $y_{\text {noise }}$ and $x_{\text {noise }}$.

\section{Path Planning Strategy}

- For Mono Directional strategy, the exact path planning for the manipulation was determined based on whenever the y coordinate of the noise object is within range of the object of interest. The operation is represented with the following algorithm's:

$$
\begin{aligned}
& x_{\text {int }}=\left\{\begin{array}{ll}
x_{\text {noises }} & \text { if } y_{\text {noise }} \max \\
x_{\text {-edges }} & \text { otherwise }
\end{array} f(x)<y_{\text {noise } \min }\right. \\
& x_{f i n}= \begin{cases}x_{\text {noise }^{x}} & \text { if } y_{\text {noise }} \text { max } \\
x_{\text {edges }} & \text { otherwise }\end{cases} \\
& y_{\text {int }}= \begin{cases}y_{\text {-edges }} & \text { if } y_{\text {noise }} \text { max } \\
y_{\text {noises }} & \text { otherwise }\end{cases} \\
& y_{f i n}= \begin{cases}y_{\text {edige }} & \text { if } y_{\text {noise }} \max \\
y_{\text {noises }} & \text { otherwise }\end{cases} \\
& \text { where } f(x)=[\text { Yobjint }]
\end{aligned}
$$

Given that Yobinnt is the y coordinate of the object of interest, $y_{\text {noise }}$ max is the y coordinate for the top corner of the noise object while Ynoise min is $y$ coordinate for the bottom corner of the noise object. Once calculated, $x_{\text {int }}$ and $y_{i n t}$ is the initial $x$ and $y$ coordinate of the generated path, $x_{f i n}$ and $y_{f i n}$ is the final $x$ and $y$ coordinate of the generated path, $x_{\text {edge }}$ and $y_{e d g e}$ is the $\mathrm{x}$ and $\mathrm{y}$ coordinate of the workspace with the highest possible coordinate value while $x_{-e d q e}$ and $y_{-e d g e}$ is the $\mathrm{x}$ and $\mathrm{y}$ coordinate of the workspace with the lowest possible coordinate value

For the Bi-directional strategy, an additional parameter which is the position of the manipulated pile in relativity to the centre of the workspace on top of the operation already done in (3) was factored in. The operation is represented with the following algorithm:

$$
\begin{aligned}
& x_{\text {int }}= \begin{cases}x_{\text {ints }} & \text { if } \text { Yhoise }_{\text {max }} \text { if } f(x) \geq 0 \\
-x_{\text {ints }} & \text { otherwise }\end{cases} \\
& x_{\text {fin }}= \begin{cases}x_{\text {final }} & \text { if } Y_{\text {noise }} \text { max } \\
-x_{\text {final }} & \text { otherwise } f(x) \geq 0\end{cases} \\
& \text { where } f(x)=x_{\text {noise }}
\end{aligned}
$$

\section{Manipulation Of Region}

- To perturb the region, the research proposes using a simple manipulation primitive (pushing motion) along a straight-line path between $x_{\text {int }} \mathrm{t}, y_{\text {int }}$ to $x_{f i n}, y_{\text {fin }}$. This is method was chosen for its applicability to a variety of other robots with or without a gripper end effector and at the same time generic enough to be able to be applied on a variety of objects.

\section{E. Repeat}

- The algorithm then repeats until the workspace is cleared of all noise object leaving the object of interest totally isolated. 


\section{Object Isolation with Minimal Impact towards the Object of Interest in a Complex Environment Using Manipulation Primitives}

Table 1: Brief summary on the differences between the designed algorithm

\begin{tabular}{|c|c|c|c|}
\hline Algorithm & $\begin{array}{c}\text { Pile Selection } \\
\text { Strategy }\end{array}$ & $\begin{array}{c}\text { Path } \\
\text { Planning } \\
\text { Strategy }\end{array}$ & $\begin{array}{c}\text { Environment } \\
\text { manipulation } \\
\text { strategy }\end{array}$ \\
\hline MSMAPPS & $\begin{array}{c}\text { Pile } \\
\text { Positioning }\end{array}$ & $\begin{array}{c}\text { Single } \\
\text { Direction }\end{array}$ & Push motion \\
\hline MSMAPOS & Pile Overlap & $\begin{array}{c}\text { Single } \\
\text { Direction }\end{array}$ & Push motion \\
\hline BSMAPOS & Pile Overlap & Bi Direction & Push motion \\
\hline
\end{tabular}

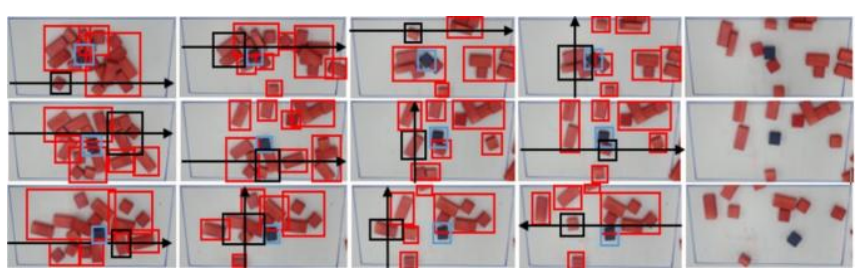

Figure 1: A visual representation of difference in operation between (top)MSMAPPS, (middle)MSMAPOS, and

(bottom)BSMAPOS for 5 iterations demonstrating the object detection (red box for noise object, blue box for object of interest), selection strategy (black box indicating selected pile), generated trajectory and direction of actuation (black line) for each method

\section{METHODOLOGY}

We implemented and experimentally evaluate the single manipulation method and its following algorithms using the Kuka Youbot which is a 5 DOF Robotic arm with a two-finger gripper as its end effector all of which is attached to a mobile platform. A camera is fitted $90 \mathrm{~cm}$ directly above the workspace at an angle of $90^{\circ}$ as seen in Figure 2(b). The camera functions to take visual information from the workspace and feed it into a computer where OpenCV is the use to discern valuable information such as the number of objects, whenever the object is noise or the object of interest as well as is spatial positioning. The operating system used to host the algorithm and its supporting functions was ROS Indigo (Robotic Operating System) operating out of Ubuntu 14.04.

For this research, we used hand made the bricks that are to be used as the object of interest as well as noise objects. The bricks were modeled after Duplo bricks hence sharing its dimensions i.e $1 \times 1$ duplo brick is $2 \mathrm{~cm} \times 2 \mathrm{~cm} \times 1 \mathrm{~cm}, 1 \mathrm{x} 2$ duplo brick is $4.5 \mathrm{~cm} \times 2 \mathrm{~cm} \times 1 \mathrm{~cm}$. The developed bricks were then spray painted red to indicate object of interest and blue to indicate noise objects. The final product can be seen in Figure 2 .

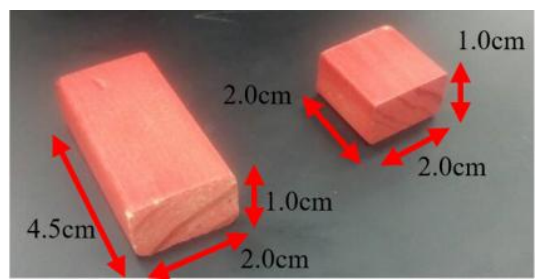

Figure 2: Dimensions of our Pseudo Duplo bricks (left 1x2, right $1 \times 1)$ which was used as object of interest (1x1) and noise objects $(1 \times 1$ and $2 \times 1)$
The experiment procedure for the simulation and actual is the same. The workspace is first set up as shown previously in Figure 3 where 18 bricks comprising of 9 of $1 \times 1$ variant and 9 $1 \times 2$ variant is randomly scattered in the field randomly but evenly around the object of interest as seen in Figure 4. The object of interest is placed in the center of the workspace. Once the scene has been set up, the program is initiated and is left to run until it returns back to home position indicating that either the object has been fully isolated or the field is now empty as seen in Figure 4. Throughout the program operation period, a camera directly above the workspace will continuously capture data in terms of the movement of the arm as well as the resulting displacement of the object of interest after each actuation. The experiment is run 3 times per designed algorithm and the results are tabulated
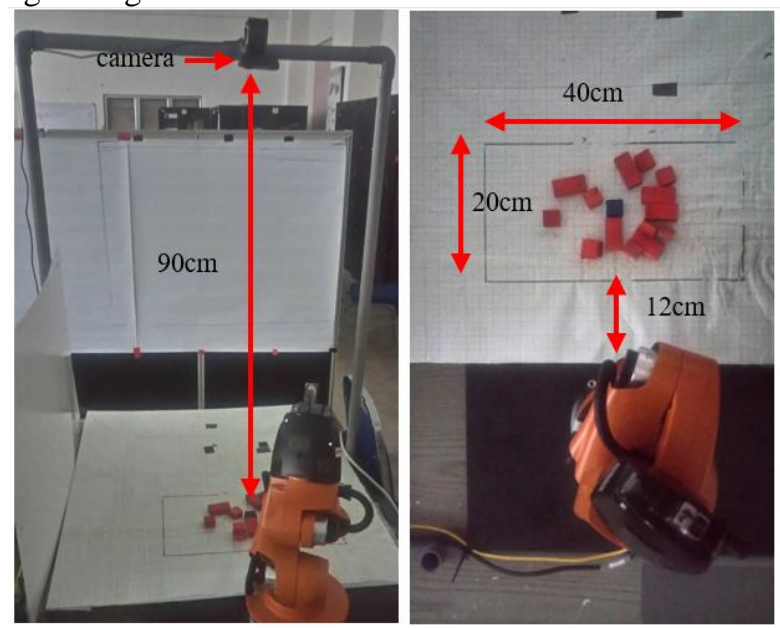

Figure 3: Full experimental setup: Kuka Youbot Robotic arm mounted on a platform with a camera overlooking the designated workspace and the pseudo duplo bricks. The task is to remove the noise objects (red) leaving only the object of interest (blue) in the workspace
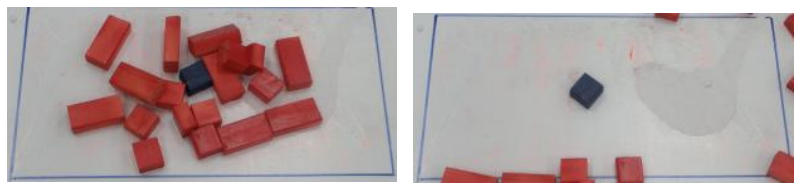

Figure 4: the difference between the field at the start of the experiment (on the left) and a field that is considered fully isolated at the end of the experiment (on the right)

\section{RESULTS AND DISCUSSION}

As mentioned previously, we have already successfully proven that the designed isolation algorithm is significantly more efficient in comparison to singulation algorithm when applied in the field of object isolation [8]. In that same research, we have successfully reduced the total number of manipulations from 1.78 manipulation per object 1.06 manipulations per object for the Mono-directional Single Manipulation Algorithm Using Pile Position Strategy (MSMAPPS), 0.78 manipulation per objects for Mono-directional Single Manipulation Algorithm Using Pile Overlap Strategy (MSMAPOS) and a further drop to 0.605 using Bi-directional Single Manipulation Algorithm Using Pile Overlap Strategy (BSMAPOS). 
This paper however is focusing on whenever using the same developed methods, we can isolate the object of interest with minimal effect towards the object of interest the same way how a precise object singulation algorithm would precisely remove each individual object per actuation with little to no affect towards its surroundings. To do this, We extrapolated the results of each algorithm from the evenly spread configurations and looked at its ability to remove the noise objects with minimal disturbance towards the object of interest. This is represented in the mapping of the movements of the object of interest throughout the isolation process as seen in Figure 5 as well as its displacement from its origin in Figure 6.

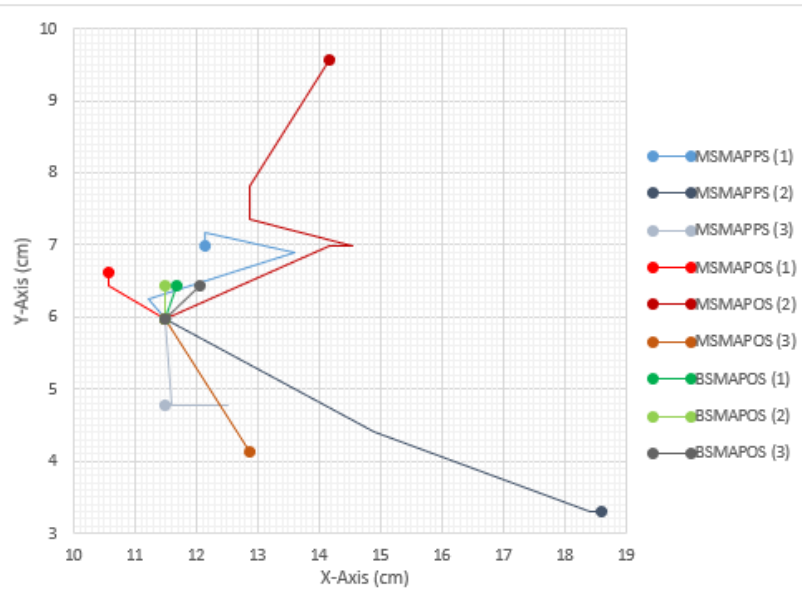

Figure 5: Chart mapping the movement of the object of interest for all 3 methods for 3 repetitions in evenly spread configuration throughout the isolation cycle. $(1$ unit $=1 \mathrm{~cm})$

It can be seen from Figure 5 and Figure 6 that both MSMAPPS and MSMAPOS have similar performance results with an average standard deviation when it comes to displacement per actuation at $0.85 \mathrm{~cm} /$ actuation and $0.75 \mathrm{~cm} /$ actuation respectively. This indicates a relatively higher impact on the object of interest when compared to the BSMAPOS which has an average of $0.27 \mathrm{~cm} /$ actuation. This incurred displacement is relatively small when compared to size of the workspace $(40 \mathrm{~cm} \times 20 \mathrm{~cm})$ or to be precise a $4.35 \%$ displacement per actuation, $3.75 \%$ displacement per actuation and $1.35 \%$ displacement per actuation respectively relative to the shortest axis on the workspace $(20 \mathrm{~cm})$. The difference in performance between BSMAPOS compared to the MSMAPPS and MSMAPOS can be attributed to the Bi Direction approach which actuates the noise objects towards the closest border rather than the Mono Direction Approach which actuates the noise objects either to the top border or the right border. By combining the Pile overlap strategy with Bi directional approach, we aim to push the largest and densest pile towards the closest edge thus not only reducing the total number of objects that is potentially displaced along the generated trajectory but also reduce the possibility of the algorithm manipulating large piles towards the object of interest rather than away as seen in Figure 7. This ultimately reduces the occurrence of the object of interest being indirectly manipulated and occasionally manipulated out of workspace as seen in MSMAPPS (2) and MSMAPOS (3) in Figure 5.

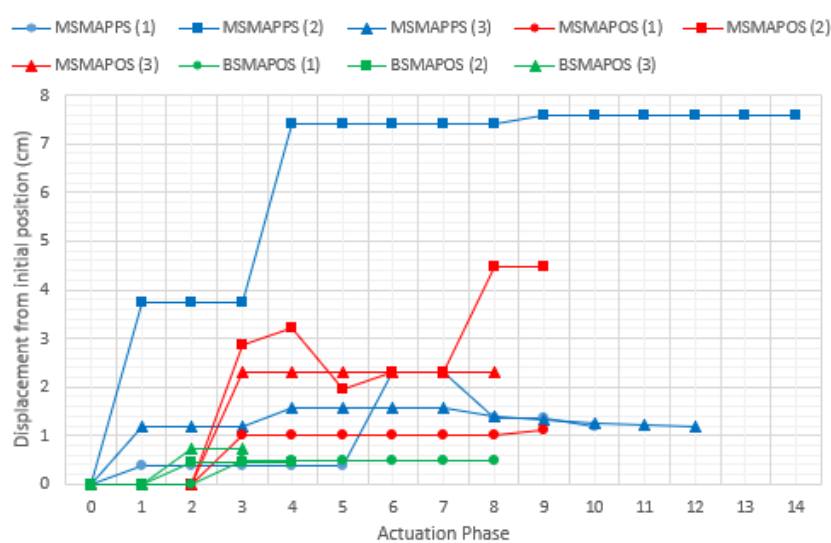

Figure 6: Performance comparison between MSMAPPS, MSMAPOS and BSMAPOS algorithm in terms of the displacement of the object of interest across the entire operation as well as the total number of manipulations required to achieve isolation

Similar to the analysis done in our previous work, we can compare the results obtained here with the work of Gupta et al [3]. We do this by assuming that Gupta's method would incur no disturbance to the object of interest as Gupta's method will precisely remove a single noise object at a time given the same situation. In this regard, while our purpose method is faster than that of Gupta's in terms of manipulation per object as discovered in past work, it loses out in terms of the disturbance incurred to the object of interest by the algorithm as it isolates the object of interest as even if the displacement is small in regards to the field, it is still large in relativity to the 0 disturbance by Gupta's method.

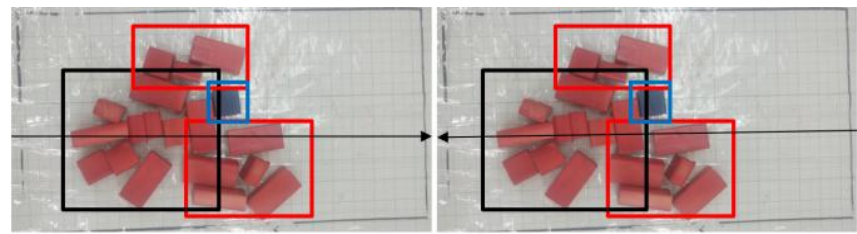

Figure 7: Comparison between the utilization of Bi directional strategy compared to mono direction strategy with Pile

Positioning Strategy when it comes to indirect manipulation.

Black box indicating selected pile, red indicating noise objects, blue indicating object of interest and black arrow line indicating the direction of propagation

\section{CONCLUSION}

This paper explored and evaluated the usage of manipulation aided perception in the context of isolating small objects (Duplo Bricks) off the Kuka Youbot workspace with minimal impact towards the object of interest. We presented three different algorithm which combines perception and manipulation to efficiently and quickly to isolate unwanted noise objects from the object of interest using a single manipulation method coupled with cluster identification. Prior result on the Kuka Youbot indicates that singulation techniques can successfully reduced the total number of manipulations from 1.78 manipulation per object to 1.06 manipulations per object for the Mono-directional Single Manipulation Algorithm Using Pile Position Strategy

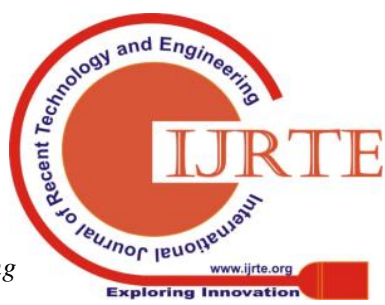




\section{Object Isolation with Minimal Impact towards the Object of Interest in a Complex Environment Using Manipulation Primitives}

(MSMAPPS), 0.78 manipulation per objects for Mono-directional Single Manipulation Algorithm Using Pile Overlap Strategy (MSMAPOS) and a further drop to 0.605 manipulation per object using Bi-directional Single Manipulation Algorithm Using Pile Overlap Strategy (BSMAPOS). Thus, in this paper, we found that the impact towards the object of interest using the same 3 developed methods are minimal with one method performing significantly better than its counterpart. MSMAPPS performed with a displacement per actuation of $0.85 \mathrm{~cm} /$ actuation, MSMAPOS at $0.75 \mathrm{~cm} /$ actuation and finally BSMAPOS performed at an average of $0.27 \mathrm{~cm} /$ actuation . These figures are relatively small in comparison to the shortest axis of the workspace or $4.35 \%$ displacement per actuation, $3.75 \%$ displacement per actuation and $1.35 \%$ displacement per actuation respectively. However, it is worth acknowledging that while the method performed with minimal impact towards the object of interest as designed, it is still a relatively high amount of actuation when compared to our benchmark gupta et al's work which would have net an average displacement of $0 \%$ per actuation due to the precise nature of singulation techniques.

\section{ACKNOWLEDGMENT}

Authors are grateful to Ministry of Higher Education Malaysia and Universiti Teknikal Malaysia Melaka (UTeM) for the financial support through UTeM PJP Grant (PJP/2018/FKE(1D)/S01600) for the publication of this research paper. This project was conducted in Center of Excellence in Robotics and Industrial Automation (CERIA) in Universiti Teknikal Malaysia Melaka.

\section{REFERENCES}

1. Y. Lin; H. Min; H. Zhou; F. Pei, "A Human-Robot-Environment Interactive Reasoning Mechanism for Object Sorting Robot," in IEEE Transactions on Cognitive and Developmental Systems, vol.PP, no.99, pp.1-1

2. S. Elliott, M. Valente and M. Cakmak, "Making objects graspable in confined environments through push and pull manipulation with a tool," 2016 IEEE International Conference on Robotics and Automation (ICRA), Stockholm, 2016, pp. 4851-4858.

3. M. Gupta, J. Müller and G. S. Sukhatme, "Using Manipulation Primitives for Object Sorting in Cluttered Environments," in IEEE Transactions on Automation Science and Engineering, vol. 12, no. 2, pp. 608-614, April 2015.

4. M. Gupta and G. S. Sukhatme, "Using manipulation primitives for brick sorting in clutter," 2012 IEEE International Conference on Robotics and Automation, Saint Paul, MN, 2012, pp. 3883-3889.

5. D. Katz, M. Kazemi, J. A. Bagnell and A. Stentz, "Clearing a pile of unknown objects using interactive perception," 2013 IEEE International Conference on Robotics and Automation, Karlsruhe, 2013, pp. 154-161.

6. L. Chang, J. R. Smith and D. Fox, "Interactive singulation of objects from a pile," 2012 IEEE International Conference on Robotics and Automation, Saint Paul, MN, 2012, pp. 3875-3882.

7. T. Maneewarn and P. Rittipravat, "Sorting objects by multiple robots using Voronoi separation and fuzzy control," Proceedings 2003 IEEE/RSJ International Conference on Intelligent Robots and Systems (IROS 2003) (Cat. No.03CH37453), 2003, pp. 2043-2048 vol.2.

8. Q.J. Shen, M.F. Miskon, K. Azha "Efficient Object Isolation in Complex Environment Using Manipulation Primitive on a Vision Based Mobile 6DOF Robotic Arm," International Journal of Mechanical and Mechatronics Engineering (IJMME),2018,pp.50-56,vol 1.

9. Y. Lin; H. Min; H. Zhou; F. Pei, "A Human-Robot-Environent Interactive Reasoning Mechanism for Object Sorting Robot," in IEEE Transactions on Cognitive and Developmental Systems, vol.PP, no.99, pp.1-1
10. S. Contreras and F. De La Rosa, "Using Deep Learning for Exploration and Recognition of Objects Based on Images," 2016 XIII Latin American Robotics Symposium and IV Brazilian Robotics Symposium (LARS/SBR), Recife, 2016, pp. 1-6

11. M. F. Miskon and R. A. Russell, "Mapping normal sensor measurement using regions," 2009 IEEE International Conference on Industrial Technology, Gippsland, VIC, 2009, pp. 1-6.

12. M. H. Jamaluddin, T. Shimono and N. Motoi, "Motion navigation in haptic bilateral system based on vision-based force compliance controller taking object coordinate into account," 2014 IEEE 23rd International Symposium on Industrial Electronics (ISIE), Istanbul, 2014, pp. 2238-2243.

13. M. H. Jamaluddin, T. Shimono and N. Motoi, "An integration method between vision-based disturbance observer and bilateral haptic system for robust tracking of target object," 2014 IEEE 13th International Workshop on Advanced Motion Control (AMC), Yokohama, 2014, pp. 723-728.

14. M. N. A. H. Sha'abani and M. F. Miskon, "The effect of anomaly detection accuracy in varying the angular resolution of sonar using Repetitive Observation Strategy," 2011 IEEE Symposium on Industrial Electronics and Applications, Langkawi, 2011, pp. 669-674.

15. Q.J. Shen (2016). Development And Analysis Of Face Recognition System On A Mobile Robot Environment (B.A Thesis, Faculty of Electrical Engineering, Universiti Teknikal Malaysia Melaka).

16.

\section{AUTHORS PROFILE}

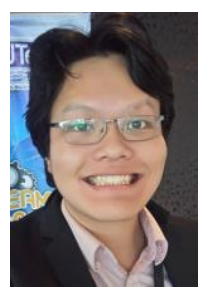

Quah Jit Shen received his Bachelors in Mechatronics Engineering from Universiti Teknikal Malaysia Melaka in 2016. He is currently pursuing his masters and subsequently PHD in mechatronics engineering at University Teknikal Malaysia Melaka.

His research focuses on designing algorithms for intelligent robotic manipulation in cluttered environment for the purpose of object isolation.

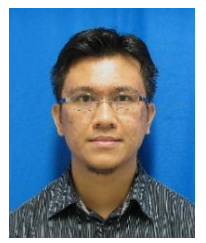

Muhammad Fahmi Miskon received his B.Eng degree in Electrical - Mechatronics Engineering from Universiti Teknologi Malaysia (UTM), in 2001. He received the M.Eng in Mechatronics from University of Newcastle Upon Tyne, in 2003 and his Ph.D in Electrical and Computer Engineering from Monash University in 2010. Currently, he is an Associate Professor at Universiti Teknikal Malaysia Melaka (UTeM) and his interests are in Robot Intelligence and novelty design.

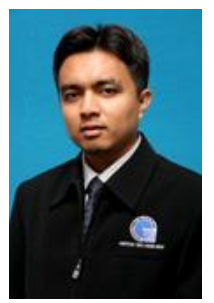

Khalil Azha Mohd Annuar received his B.Eng degree in Electrical Engineering (Electronic) from Universiti Teknologi Malaysia (UTM), in 2006. He received the M.Eng degree in Mechatronics and Automatic Control engineering also from UTM, in 2014. Currently, he is a Lecturer at Universiti Teknikal Malaysia Melaka (UTeM) and his interests are in control system, artificial intelligent and energy management. 